TRANSACTIONS OF THE

AMERICAN MATHEMATICAL SOCIETY

Volume 365, Number 7, July 2013, Pages 3477-3492

S 0002-9947(2012)05769-2

Article electronically published on December 17, 2012

\title{
UNIFORM ESTIMATES FOR THE LOCAL RESTRICTION OF THE FOURIER TRANSFORM TO CURVES
}

\author{
SPYRIDON DENDRINOS AND DETLEF MÜLLER
}

\begin{abstract}
We prove sharp estimates, with respect to the affine arclength measure, for the restriction of the Fourier transform to a class of curves in $\mathbb{R}^{d}$ that includes curves of finite type. This measure possesses certain invariance and mitigation properties which are important in establishing uniform results.
\end{abstract}

\section{Introduction AND STATEMENT OF THE MAIN RESUlt}

There has recently been considerable attention to problems in Euclidean harmonic analysis where the underlying arclength or surface measure is chosen to be the so-called affine arclength or surface measure respectively (see [7] and the references therein). The objective is to obtain global and universal estimates, i.e., estimates where the boundedness properties are uniform over large classes of curves or surfaces. In the case of the problem of local Fourier restriction to curves, with which this article is concerned, these estimates can be used to deduce corresponding ones with respect to the Riemannian arclength measure (see [10] for a derivation of results with Riemannian arclength from results with affine arclength and 11. for a discussion). For global estimates, in general one does not expect to obtain any results using the Riemannian arclength measure and it seems that the affine arclength is the natural measure to use. Moreover, these have many interesting applications (see e.g. [6] and, for a connection to an affine isoperimetric inequality, [12]).

In the case of curves $\gamma: I \rightarrow \mathbb{R}^{d}, I \subseteq \mathbb{R}$, the affine arclength measure $d \sigma$ is defined by its action on a test function $\phi$ as

$$
d \sigma(\phi)=\int_{I} \phi(\gamma(t))\left|L_{\gamma}(t)\right|^{\frac{2}{d(d+1)}} d t
$$

where

$$
L_{\gamma}(t)=\operatorname{det}\left(\gamma^{\prime}(t), \ldots, \gamma^{(d)}(t)\right) .
$$

Notice that, like the Riemannian arclength, this measure is invariant under reparametrisations of the curve $\gamma$. The Fourier restriction estimates that one ultimately hopes to obtain take the form

$$
\int_{I}|\widehat{f}(\gamma(t))|^{q}\left|L_{\gamma}(t)\right|^{\frac{2}{d(d+1)}} d t \leq C\|f\|_{L^{p}\left(\mathbb{R}^{d}\right)}^{q},
$$

Received by the editors June 15, 2011.

2010 Mathematics Subject Classification. Primary 42B10.

This research was partly carried out during a visit of the first author to the University of Kiel, funded by the Deutscher Akademischer Austausch Dienst (DAAD). 
for some choice of $p, q$, and $C$ and for all Schwartz functions $f$ and intervals $I \subseteq \mathbb{R}$. In addition, the choice of $p, q, C$ and $I$ should be uniform over a large class of curves $\gamma$.

This problem was first considered by Sjölin [13 for $d=2$ where he showed that (11) holds uniformly for all curves $\gamma$ and intervals $I$ such that $L_{\gamma}$ stays single-signed on $I$, exponents $p$ and $q$ satisfying $p^{\prime}=3 q$ (where $1 / p+1 / p^{\prime}=1$ ) and $1 \leq p<4 / 3$, and a constant $C$ that depends only on $p$. He also gave a counterexample of a rapidly oscillating curve $\gamma$ where (1) fails. Regarding higher dimensions, and in order to establish what should be the necessary and sufficient conditions on $p$ and $q$, the nondegenerate curve $\gamma(t)=\left(t, t^{2}, \ldots, t^{d}\right)$ has been considered. For this curve, for which $L_{\gamma}$ is equal to a constant, in the seminal article [8] Drury showed that the conditions

$$
p^{\prime}=\frac{d(d+1)}{2} q \quad \text { and } \quad 1 \leq p<\frac{d^{2}+d+2}{d^{2}+d}
$$

are sufficient for (1) to hold. The necessity of the first condition in (2) can be shown by a simple scaling argument, should one require the Fourier restriction result to be global, i.e., true for all intervals $I \subseteq \mathbb{R}$. The necessity of the second condition in (2) follows from the work of Arkhipov, Chubarikov, and Karatsuba [1. There followed a series of articles by Drury and Marshall [10, 11, 9] which aimed at proving a local Fourier restriction result, allowing $I$ to depend on $\gamma$, for which, nevertheless, the $L^{p} \rightarrow L^{q}$ mapping properties are uniform over a family of curves and which includes all curves of finite type.

Unfortunately, even though these articles have introduced many important new ideas to this circle of problems, in particular a general strategy based on the method of offspring curves together with the use of two key geometric inequalities in the proofs, it appears to us that the argument in Section 2 of [11, in particular in the derivation of the geometric inequality in Theorem 3 for the case of perturbed exponentials from the unperturbed case, is not conclusive. Indeed, if one tries to complete the proof by isolating the leading term of pure exponentials and estimating the remaining terms using Lemma 5 in 11, then the corresponding matrices will not all be of the type described in the lemma, as simple examples show (e.g. consider $\gamma(t)=\left(t, t^{2}+t^{3}\right)$ for $t$ near the origin). This problem affects all the perturbed results and, as a consequence, only the proofs for the results on curves of the precise form $\gamma(t)=\left(t^{a_{1}}, \ldots, t^{a_{d}}\right)$, where $t \geq 0$ and the $a_{i}$ are real, and $p$ is from a restricted $p$ range, appear to be completely rigorous. This case was generalised to the full range of $p$ 's given in (2) by the recent work of Bak, Oberlin, and Seeger [2] for the above case of pure monomials.

The purpose of the present article is to prove a local uniform Fourier restriction estimate on the critical line for the perturbed case and the full range of $p$ 's given in (2), thereby concluding the original line of pursuit of Drury and Marshall. We resolve the problem that occurred in [1] by treating both the unperturbed and perturbed cases simultaneously and using a formula that first appeared in [4 and was also used in [7. This formula is described after Proposition 5 below. At the same time, our method gives an alternative proof even for the pure monomial case which, due to its similarities to the methods in [7, should be more relevant for future work on global uniform estimates for Fourier restriction to general classes of curves containing mixed homogeneities. 
Theorem 1. Let $\Gamma(t)=\left(t^{a_{1}} \theta_{1}(t), \ldots, t^{a_{d}} \theta_{d}(t)\right), t \geq 0$, where, for each $1 \leq i \leq d$, $a_{i} \in \mathbb{R} \backslash\{0\}, a_{i}$ are distinct, $\theta_{i}$ are real-valued and in $C^{d}(\mathbb{R})$, the limits $\lim _{t \rightarrow 0} \bar{\theta}_{i}(t)$ exist and are not equal to 0 , and for all $1 \leq m \leq d$,

$$
\lim _{t \rightarrow 0} t^{m} \theta_{i}^{(m)}(t)=0 \text {. }
$$

Then there exist $C$, only depending on $d$ and $p$, and $\delta$, depending on the $a_{i}$, the $\theta_{i}$ and $d$, such that

$$
\int_{0}^{\delta}|\widehat{f}(\Gamma(t))|^{q}\left|L_{\Gamma}(t)\right|^{\frac{2}{d(d+1)}} d t \leq C\|f\|_{p}^{q}
$$

for all Schwartz functions $f$, where $p$ and $q$ satisfy (2). In the particular case where, for all $1 \leq i \leq d$, the $\theta_{i}$ are identically constant, $\delta$ can be taken to be equal to $\infty$.

Remark 1. Inequality (3) is invariant under reparametrisations of $\Gamma$ and this is exploited in the proof by using an exponential parametrisation.

Remark 2. Inequality (3) is invariant under replacement of $\Gamma$ by $A \Gamma$ for any invertible affine transformation $A$.

Remark 3. Curves of finite type at the origin, i.e. those whose derivatives at the origin do not vanish to infinite order, satisfy the conditions of the theorem with $a_{i} \in \mathbb{N}$ after a suitable affine transformation.

Remark 4. The global result $(\delta=\infty)$, for the particular case where the $\theta_{i}$ are identically constant, follows from the local result by nonisotropic scaling using the dilations $x \mapsto\left(\lambda^{a_{1}} x_{1}, \ldots, \lambda^{a_{d}} x_{d}\right)$.

Structure of the article. Section 2 contains the key geometric inequalities for the original curve and its first order offspring curves. The generalisation in Section 3 to offspring curves of higher orders is needed in order to define the classes of curves $\mathcal{K}_{T}$ that appear in Section 4 Section 4 contains the main line of the proof, which heavily draws on ideas from Bak, Oberlin, and Seeger [2].

Notation. We explicitly display the dependencies of the various constants by means of subscripts. We do however suppress the explicit display of the dependence on the dimension $d$, in order to slightly ease notation. The precise values of the various constants called " $C$ " may change from line to line. Finally, by $A \lesssim B$, we mean that there exists a positive constant $C$, only dependent on $d$, such that $|A| \leq C|B|$ and, by $A \sim B$, that $A \lesssim B \lesssim A$.

\section{OfFSPRING CURVES AND GEOMETRIC INEQUALITIES}

Our proof of Theorem 1 is based on the method of offspring curves, which was introduced by Drury 8, together with two geometric inequalities, which were introduced in Drury and Marshall 10 as the key ingredients for this method. These are contained in Propositions 5 and 8 in this section.

We start by utilising the reparametrisation invariance of (3) and replace $t$ by $e^{-t}$ so that hereafter we may assume that the curve $\Gamma$ takes the form

$$
\Gamma(t)=\left(\Gamma_{1}(t), \ldots, \Gamma_{d}(t)\right)=\left(e^{a_{1} t} \psi_{1}(t), \ldots, e^{a_{d} t} \psi_{d}(t)\right),
$$

with new $a_{i}$ 's which arise from the original ones by a change of signs. Permuting the coordinates, if necessary, we may and shall also assume that $a_{1}<\ldots<a_{d}$. For each 
$1 \leq i \leq d, \psi_{i}(\infty):=\lim _{t \rightarrow \infty} \psi_{i}(t)$ exists and is nonzero and $\lim _{t \rightarrow \infty} \psi_{i}^{(m)}(t)=0$ for all $1 \leq m \leq d$.

We first need an estimate for the minors of the determinant

$$
L_{\Gamma}(t)=\operatorname{det}\left(\Gamma^{\prime}(t), \ldots, \Gamma^{(d)}(t)\right),
$$

which we denote by

$$
L_{\Gamma_{1} \cdots \Gamma_{m}}(t)=\left|\begin{array}{ccc}
\Gamma_{1}^{\prime}(t) & \cdots & \Gamma_{1}^{(m)}(t) \\
\vdots & & \vdots \\
\Gamma_{m}^{\prime}(t) & \cdots & \Gamma_{m}^{(m)}(t)
\end{array}\right|
$$

for $1 \leq m \leq d$.

Lemma 2. Let $1 \leq m \leq d$. Then there exists $T$, depending on $d$, the $a_{i}$, and the $\psi_{i}$, such that

$$
L_{\Gamma_{1} \cdots \Gamma_{m}}(t) \sim e^{t \sum_{i=1}^{m} a_{i}} \prod_{i=1}^{m}\left(a_{i} \psi_{i}(\infty)\right) \prod_{1 \leq i<j \leq m}\left(a_{j}-a_{i}\right)
$$

for all $t>T$.

Proof.

$$
\begin{aligned}
L_{\Gamma_{1} \cdots \Gamma_{m}}(t)=\left|\begin{array}{ccc}
e^{a_{1} t}\left(a_{1} \psi_{1}(t)+\psi_{1}^{\prime}(t)\right) & \cdots & e^{a_{1} t}\left(\sum_{k=0}^{m}\left(\begin{array}{c}
m \\
k
\end{array}\right) a_{1}^{k} \psi_{1}^{(m-k)}(t)\right) \\
\vdots & \vdots \\
e^{a_{m} t}\left(a_{m} \psi_{m}(t)+\psi_{m}^{\prime}(t)\right) & \cdots & e^{a_{m} t}\left(\sum_{k=0}^{m}\left(\begin{array}{c}
m \\
k
\end{array}\right) a_{m}^{k} \psi_{m}^{(m-k)}(t)\right)
\end{array}\right| \\
=e^{t \sum_{i=1}^{m} a_{i}} \prod_{i=1}^{m} \psi_{i}(t)\left|\begin{array}{ccc}
a_{1}+\frac{\psi_{1}^{\prime}(t)}{\psi_{1}(t)} & \cdots & a_{1}^{m}+\sum_{k=0}^{m-1}\left(\begin{array}{c}
m \\
k
\end{array}\right) a_{1}^{k} \frac{\psi_{1}^{(m-k)}(t)}{\psi_{1}(t)} \\
\vdots & & \vdots \\
a_{m}+\frac{\psi_{m}^{\prime}(t)}{\psi_{m}(t)} & \cdots & a_{m}^{m}+\sum_{k=0}^{m-1}\left(\begin{array}{c}
m \\
k
\end{array}\right) a_{m}^{k} \frac{\psi_{m}^{(m-k)}(t)}{\psi_{m}(t)}
\end{array}\right|
\end{aligned}
$$

Given any $\epsilon>0$, there exists some $T=T\left(\epsilon, \psi_{1}, \ldots, \psi_{m}\right)$ such that

$$
\sup _{1 \leq l \leq m}\left|\psi_{i}^{(l)}(t)\right|<\epsilon \quad \text { and } \quad \psi_{i}(t) \sim \psi_{i}(\infty)
$$

for all $t>T$ and $1 \leq i \leq m$. Therefore, using the multilinearity of the determinant, expanding out each term in the last determinant except for the leading term,

$$
\left|\begin{array}{ccc}
a_{1} & \cdots & a_{1}^{m} \\
\vdots & & \vdots \\
a_{m} & \cdots & a_{m}^{m}
\end{array}\right|
$$

and choosing $\epsilon=\epsilon\left(a_{1}, \ldots, a_{m}, \psi_{1}, \ldots, \psi_{m}, m\right)$ sufficiently small, we obtain the estimate

$$
\begin{aligned}
L_{\Gamma_{1} \cdots \Gamma_{m}}(t) & \sim e^{t \sum_{i=1}^{m} a_{i}} \prod_{i=1}^{m} \psi_{i}(\infty)\left|\begin{array}{ccc}
a_{1} & \cdots & a_{1}^{m} \\
\vdots & & \vdots \\
a_{m} & \cdots & a_{m}^{m}
\end{array}\right| \\
& =e^{t \sum_{i=1}^{m} a_{i}} \prod_{i=1}^{m}\left(a_{i} \psi_{i}(\infty)\right) \prod_{1 \leq i<j \leq m}\left(a_{j}-a_{i}\right) .
\end{aligned}
$$


Definition 3. We define the family of offspring curves of $\Gamma$ by

$$
\Gamma_{h}(t)=\sum_{j=1}^{d} \Gamma\left(t+h_{j}\right)
$$

where the parameter $h=\left(h_{1}, \ldots, h_{d}\right) \in \mathbb{R}^{d}$ satisfies $0=h_{1} \leq h_{2} \leq \ldots \leq h_{d}$.

A useful property of the exponential parametrisation of our original curve $\Gamma$ is that any offspring curve of $\Gamma$ resembles $\Gamma$ as is made precise in the discussion in the beginning of Section 5. This is the reason for the similarities between Lemma 2 and the following.

Lemma 4. Let $\Gamma_{h}$ be an offspring curve of $\Gamma$. Then there exists $T$, depending on $d$, the $a_{i}$, and the $\psi_{i}$, such that

$$
L_{\Gamma_{h}}(t) \sim e^{t \sum_{i=1}^{d} a_{i}} \prod_{i=1}^{d}\left(a_{i} \psi_{i}(\infty) \sum_{j=1}^{d} e^{a_{i} h_{j}}\right) \prod_{1 \leq i<j \leq d}\left(a_{j}-a_{i}\right)
$$

for all $t>T$.

Proof.

$$
\begin{aligned}
& L_{\Gamma_{h}}(t)=\mid \begin{array}{cc}
e^{a_{1} t} \sum_{j=1}^{d} e^{a_{1} h_{j}}\left(a_{1} \psi_{1}\left(t+h_{j}\right)+\psi_{1}^{\prime}\left(t+h_{j}\right)\right) & \ldots \\
\vdots & \\
e^{a_{d} t} \sum_{j=1}^{d} e^{a_{d} h_{j}}\left(a_{d} \psi_{d}\left(t+h_{j}\right)+\psi_{d}^{\prime}\left(t+h_{j}\right)\right) & \ldots
\end{array} \\
& \cdots \quad e^{a_{1} t} \sum_{j=1}^{d} e^{a_{1} h_{j}}\left(\sum_{k=0}^{d}\left(\begin{array}{l}
d \\
k
\end{array}\right) a_{1}^{k} \psi_{1}^{(d-k)}\left(t+h_{j}\right)\right) \\
& \cdots \quad e^{a_{d} t} \sum_{j=1}^{d} e^{a_{d} h_{j}}\left(\sum_{k=0}^{d}\left(\begin{array}{l}
d \\
k
\end{array}\right) a_{d}^{k} \psi_{d}^{(d-k)}\left(t+h_{j}\right)\right) \\
& =e^{t \sum_{i=1}^{d} a_{i}} \prod_{i=1}^{d}\left(\sum_{j=1}^{d} e^{a_{i} h_{j}} \psi_{i}\left(t+h_{j}\right)\right) \\
& \times\left|\begin{array}{ccc}
a_{1}+\frac{\sum_{j=1}^{d} e^{a_{1} h_{j}} \psi_{1}^{\prime}\left(t+h_{j}\right)}{\sum_{j=1}^{d} e^{a_{1} h_{j}} \psi_{1}\left(t+h_{j}\right)} & \cdots & a_{1}^{d}+\frac{\sum_{k=0}^{d-1}\left(\begin{array}{c}
d \\
k
\end{array}\right) a_{1}^{k}\left(\sum_{j=1}^{d} e^{a_{1} h_{j}} \psi_{1}^{(d-k)}\left(t+h_{j}\right)\right)}{\sum_{j=1}^{d} e^{a_{1} h_{j}} \psi_{1}\left(t+h_{j}\right)} \\
\vdots & & \vdots \\
a_{d}+\frac{\sum_{j=1}^{d} e^{a_{d} h_{j}} \psi_{d}^{\prime}\left(t+h_{j}\right)}{\sum_{j=1}^{d} e^{a^{h} h_{j}} \psi_{d}\left(t+h_{j}\right)} & \cdots & a_{d}^{d}+\frac{\sum_{k=0}^{d-1}\left(\begin{array}{c}
d \\
k
\end{array}\right) a_{d}^{k}\left(\sum_{j=1}^{d} e^{a_{d} h_{j}} \psi_{d}^{(d-k)}\left(t+h_{j}\right)\right)}{\sum_{j=1}^{d} e^{a^{h^{h}}} \psi_{d}\left(t+h_{j}\right)}
\end{array}\right| .
\end{aligned}
$$

As in Lemma 2 we now use the fact that, given any $\epsilon>0$, there exists some $T=T\left(\epsilon, \psi_{1}, \ldots, \psi_{d}\right)$ such that

$$
\sup _{1 \leq l \leq d}\left|\psi_{i}^{(l)}(t)\right|<\epsilon \quad \text { and } \quad \psi_{i}(t) \sim \psi_{i}(\infty)
$$

for all $t>T$ and $1 \leq i \leq d$. We recall that all the $h_{j}$ are nonnegative. Therefore, the same estimates hold for each $\psi_{i}\left(t+h_{j}\right)$ as for $\psi_{i}(t)$. Using the multilinearity of the determinant, expanding out all terms except for the leading term

$$
\left|\begin{array}{ccc}
a_{1} & \cdots & a_{1}^{d} \\
\vdots & & \vdots \\
a_{d} & \cdots & a_{d}^{d}
\end{array}\right|
$$


and choosing $\epsilon=\epsilon\left(a_{1}, \ldots, a_{d}, \psi_{1}, \ldots, \psi_{d}, d\right)$ sufficiently small (note the independence on the $h_{j}$ ), we obtain the required estimate

$$
L_{\Gamma_{h}}(t) \sim e^{t \sum_{i=1}^{d} a_{i}} \prod_{i=1}^{d}\left(a_{i} \psi_{i}(\infty) \sum_{j=1}^{d} e^{a_{i} h_{j}}\right) \prod_{1 \leq i<j \leq d}\left(a_{j}-a_{i}\right),
$$

concluding the proof of Lemma 4 .

Lemmas 2 (for $m=d$ ) and 4 lead to the first geometric inequality.

Proposition 5. Let $T$ be such that the estimates of Lemmas 2 and 4 hold. Then

$$
L_{\Gamma_{h}}(t) \gtrsim \prod_{j=1}^{d}\left|L_{\Gamma}\left(t+h_{j}\right)\right|^{\frac{1}{d}}
$$

for all $t>T$.

Proof. Let $t>T$. Then

$$
\begin{aligned}
L_{\Gamma_{h}}(t) & \sim e^{t \sum_{i=1}^{d} a_{i}} \prod_{i=1}^{d}\left(a_{i} \psi_{i}(\infty) \sum_{j=1}^{d} e^{a_{i} h_{j}}\right) \prod_{1 \leq i<j \leq d}\left(a_{j}-a_{i}\right) \\
& \gtrsim e^{t \sum_{i=1}^{d} a_{i}} \prod_{i=1}^{d}\left(a_{i} \psi_{i}(\infty) e^{\frac{a_{i}}{d} \sum_{j=1}^{d} h_{j}}\right) \prod_{1 \leq i<j \leq d}\left(a_{j}-a_{i}\right) \\
& =e^{\frac{1}{d}\left(\sum_{i=1}^{d} a_{i}\right)\left(\sum_{j=1}^{d} t+h_{j}\right)} \prod_{i=1}^{d}\left(a_{i} \psi_{i}(\infty)\right) \prod_{1 \leq i<j \leq d}\left(a_{j}-a_{i}\right) \\
& \sim \prod_{j=1}^{d}\left|L_{\Gamma}\left(t+h_{j}\right)\right|^{\frac{1}{d}} .
\end{aligned}
$$

Here, we have used Lemmas 2 and 4, together with the inequality relating the arithmetic and geometric means of the quantities $e^{a_{i} h_{j}}$.

The second geometric inequality concerns the Jacobian $J_{\Phi_{\Gamma}}$ of the mapping

$$
\Phi_{\Gamma}\left(t_{1}, \ldots, t_{d}\right)=\sum_{i=1}^{d} \Gamma\left(t_{i}\right),
$$

where $T<t_{1}<\ldots<t_{d}$ and $T$ is large enough, in terms of its relation with the function $L_{\Gamma}$. For this purpose, a formula linking $J_{\Phi_{\Gamma}}$ with the minors of $L_{\Gamma}$ was established in 44 (this formula is also presented in [7). We note that although this formula was first established for polynomial curves $\Gamma$, it is also true more generally, and with precisely the same proof, for any curve $\Gamma$ and any open interval $I$ as long as every minor of $L_{\Gamma}$ does not vanish and stays single-signed on $I$. This is true for our curve $\Gamma$ by Lemma 2 . The formula expresses $J_{\Phi_{\Gamma}}$ as a series of nested integrals in the following way. We define inductively a sequence of multivariate functions $I_{r}$, $1 \leq r \leq d$. Each $I_{r}$ will be defined on $(T, \infty)^{r}$, with $T$ chosen large enough as in Lemma 2. For $r=1$ we set

$$
I_{1}(x)=\frac{L_{\Gamma_{1} \ldots \Gamma_{d-2}}(x) L_{\Gamma}(x)}{L_{\Gamma_{1} \ldots \Gamma_{d-1}}(x)^{2}}
$$


and then inductively

$$
\begin{aligned}
& I_{r}\left(x_{1}, \ldots, x_{r}\right) \\
& \quad=\prod_{s=1}^{r} \frac{L_{\Gamma_{1} \ldots \Gamma_{d-r-1}}\left(x_{s}\right) L_{\Gamma_{1} \ldots \Gamma_{d-r+1}}\left(x_{s}\right)}{L_{\Gamma_{1} \ldots \Gamma_{d-r}}\left(x_{s}\right)^{2}} \int_{x_{1}}^{x_{2}} d y_{1} \ldots \int_{x_{r-1}}^{x_{r}} d y_{r-1} I_{r-1}\left(y_{1}, \ldots, y_{r-1}\right),
\end{aligned}
$$

where, in order to make sense of $I_{d-1}$ and $I_{d}$, we set $L_{\Gamma_{1} \ldots \Gamma_{r}} \equiv 1$ for $r=-1,0$. We have

$$
J_{\Phi_{\Gamma}}\left(t_{1}, \ldots, t_{d}\right)=I_{d}\left(t_{1}, \ldots, t_{d}\right)
$$

for $T<t_{1}<\ldots<t_{d}$.

In order to use (5) we first need the following elementary lemma and its corollary.

Lemma 6. Let $x_{1}<\ldots<x_{k-1}<w<z<x_{k+1}<\ldots<x_{l}$ and $0<\eta<1$. Then

$$
\int_{w}^{z} e^{x_{k}} \prod_{1 \leq i<j \leq l}\left(x_{j}-x_{i}\right) d x_{k} \geq C_{\eta} e^{w+\eta(z-w)} \int_{w}^{z} \prod_{1 \leq i<j \leq l}\left(x_{j}-x_{i}\right) d x_{k} .
$$

Proof. There exist integers $k_{1} \in[0, k-1]$ and $k_{2} \in[k+1, l+1]$ such that

$$
z-x_{k_{1}} \leq 2\left(w-x_{k_{1}}\right),
$$

for all integers $j \in\left(k_{1}, k-1\right]$

$$
\begin{gathered}
z-x_{j}>2\left(w-x_{j}\right), \\
x_{k_{2}}-w \leq 2\left(x_{k_{2}}-z\right),
\end{gathered}
$$

and for all integers $j \in\left[k+1, k_{2}\right)$

$$
x_{j}-w>2\left(x_{j}-z\right) .
$$

Here, we use the convention that if $k_{1}=0$, then (7) is ignored, if $k_{1}=k-1$, then (8) is ignored, if $k_{2}=k+1$, then (10) is ignored, and if $k_{2}=l+1$, then (9) is ignored. All possible configurations of the $x_{i}, w, z$ will satisfy relations (7) - (10) for some $k_{1}, k_{2}$. One may check that if there exists an integer $k_{1} \in[1, k-1]$ such that (77) holds, then $z-x_{j} \leq 2\left(w-x_{j}\right)$ for all integers $j \in\left[1, k_{1}\right]$. Similarly, if there exists an integer $k_{2} \in[k+1, l]$ such that (9) holds, then $x_{j}-w \leq 2\left(x_{j}-z\right)$ for all integers $j \in\left[k_{2}, l\right]$. To be more precise on how we choose $k_{1}$ and $k_{2}$, if $z+x_{1}>2 w$, we choose $k_{1}=0$; otherwise, we choose $1 \leq k_{1} \leq k-1$ maximal such that $z+x_{k_{1}} \leq 2 w$. Similarly, if $x_{l}<2 z-w$, then we choose $k_{2}:=l+1$; otherwise, we choose $k_{2} \geq k+1$ minimal with $x_{k_{2}} \geq 2 z-w$.

Let

$$
I=\int_{w}^{z} e^{x_{k}} \prod_{1 \leq j<k}\left(x_{k}-x_{j}\right) \prod_{k<j \leq l}\left(x_{j}-x_{k}\right) d x_{k},
$$

$\Delta=z-w$, and choose some $\eta^{\prime}$ satisfying $0<\eta<\eta^{\prime}<1$. We have

$$
I \geq \int_{w+\eta \Delta}^{w+\eta^{\prime} \Delta} e^{x_{k}} \prod_{1 \leq j<k}\left(x_{k}-x_{j}\right) \prod_{k<j \leq l}\left(x_{j}-x_{k}\right) d x_{k} .
$$

For all $x_{k} \in(w, z)$ and all integers $j \in\left[1, k_{1}\right]$,

$$
x_{k}-x_{j} \geq w-x_{j} \geq \frac{1}{2}\left(z-x_{j}\right) \geq \frac{1}{2}\left(x_{k}-x_{j}\right),
$$


and for all $x_{k} \in(w, z)$ and all integers $j \in\left[k_{2}, l\right]$,

$$
x_{j}-x_{k} \geq x_{j}-z \geq \frac{1}{2}\left(x_{j}-w\right) \geq \frac{1}{2}\left(x_{j}-x_{k}\right) .
$$

Hence

$$
\begin{aligned}
I \gtrsim & \prod_{1 \leq j \leq k_{1}}\left(z-x_{j}\right) \prod_{k_{2} \leq j \leq l}\left(x_{j}-w\right) \int_{w+\eta \Delta}^{w+\eta^{\prime} \Delta} e^{x_{k}} \prod_{k_{1}<j<k}\left(x_{k}-x_{j}\right) \prod_{k<j<k_{2}}\left(x_{j}-x_{k}\right) d x_{k} \\
\geq & \prod_{1 \leq j \leq k_{1}}\left(z-x_{j}\right) \prod_{k_{2} \leq j \leq l}\left(x_{j}-w\right) \prod_{k_{1}<j<k}\left(w+\eta \Delta-x_{j}\right) \prod_{k<j<k_{2}}\left(x_{j}-w-\eta^{\prime} \Delta\right) \\
& \times \int_{w+\eta \Delta}^{w+\eta^{\prime} \Delta} e^{x_{k}} d x_{k} .
\end{aligned}
$$

We also have for all integers $j \in\left(k_{1}, k\right)$, by (7),

$$
w+\eta \Delta-x_{j}=z-x_{j}-(1-\eta) \Delta \geq \eta \Delta>\frac{1}{2} \eta\left(z-x_{j}\right) \geq \frac{1}{2} \eta\left(x_{k}-x_{j}\right),
$$

and for all integers $j \in\left(k, k_{2}\right)$, by (9),

$$
x_{j}-w-\eta^{\prime} \Delta \geq\left(1-\eta^{\prime}\right) \Delta>\frac{1}{2}\left(1-\eta^{\prime}\right)\left(x_{j}-w\right) \geq \frac{1}{2}\left(1-\eta^{\prime}\right)\left(x_{j}-x_{k}\right),
$$

for any $x_{k} \in(w, z)$. Hence

$$
\begin{aligned}
I \gtrsim & \eta^{k-k_{1}-1}\left(1-\eta^{\prime}\right)^{k_{2}-k-1} \Delta^{k_{2}-k_{1}-2} e^{w+\eta \Delta}\left(e^{\left(\eta^{\prime}-\eta\right) \Delta}-1\right) \\
& \times \prod_{1 \leq j \leq k_{1}}\left(z-x_{j}\right) \prod_{k_{2} \leq j \leq l}\left(x_{j}-w\right) \\
\geq & \eta^{k-k_{1}-1}\left(1-\eta^{\prime}\right)^{k_{2}-k-1}\left(\eta^{\prime}-\eta\right) \Delta^{k_{2}-k_{1}-1} e^{w+\eta \Delta} \prod_{1 \leq j \leq k_{1}}\left(z-x_{j}\right) \prod_{k_{2} \leq j \leq l}\left(x_{j}-w\right) \\
\gtrsim & \eta^{k-k_{1}-1}\left(1-\eta^{\prime}\right)^{k_{2}-k-1}\left(\eta^{\prime}-\eta\right) e^{w+\eta \Delta} \int_{w}^{z} \prod_{1 \leq j<k}\left(x_{k}-x_{j}\right) \prod_{k<j \leq l}\left(x_{j}-x_{k}\right) d x_{k},
\end{aligned}
$$

which easily implies (6), since the rest of the terms in the Vandermonde products on both sides of (6) do not depend on the variable of integration $x_{k}$.

Corollary 7. Let $t_{1}<\ldots<t_{l}$ and $a>0$. Then

$\int_{t_{1}}^{t_{2}} d x_{1} \ldots \int_{t_{l-1}}^{t_{l}} d x_{l-1} e^{a \sum_{i=1}^{l-1} x_{i}} \prod_{1 \leq i<j \leq l-1}\left(x_{j}-x_{i}\right) \gtrsim e^{\frac{a(l-1)}{l} \sum_{i=1}^{l} t_{i}} \prod_{1 \leq i<j \leq l}\left(t_{j}-t_{i}\right)$.

Proof. We may apply Lemma $6 l-1$ times with any $\eta_{i} \in(0,1), 1 \leq i \leq l-1$, to obtain

$$
\begin{aligned}
& \int_{t_{1}}^{t_{2}} d x_{1} \ldots \int_{t_{l-1}}^{t_{l}} d x_{l-1} e^{\sum_{i=1}^{l-1} x_{i}} \prod_{1 \leq i<j \leq l-1}\left(x_{j}-x_{i}\right) \\
& \gtrsim e^{\sum_{i=1}^{l-1} \eta_{i} t_{i}+\left(1-\eta_{i}\right) t_{i+1}} \int_{t_{1}}^{t_{2}} d x_{1} \ldots \int_{t_{l-1}}^{t_{l}} d x_{l-1} \prod_{1 \leq i<j \leq l-1}\left(x_{j}-x_{i}\right) \\
& \gtrsim e^{\sum_{i=1}^{l-1} \eta_{i} t_{i}+\left(1-\eta_{i}\right) t_{i+1}} \prod_{1 \leq i<j \leq l}\left(t_{j}-t_{i}\right)
\end{aligned}
$$


(for a proof of the last inequality, see e.g. [5, Lemma 4.4]). If, in particular, we choose $\eta_{i}=1-i / l$, we have

$$
\int_{t_{1}}^{t_{2}} d x_{1} \ldots \int_{t_{l-1}}^{t_{l}} d x_{l-1} e^{\sum_{i=1}^{l-1} x_{i}} \prod_{1 \leq i<j \leq l-1}\left(x_{j}-x_{i}\right) \gtrsim e^{\frac{l-1}{l} \sum_{i=1}^{l} t_{i}} \prod_{1 \leq i<j \leq l}\left(t_{j}-t_{i}\right) .
$$

A simple change of variables, $x_{i} \mapsto a x_{i}$ for all $1 \leq i \leq l$, yields the required estimate.

We are now ready to state and prove the second geometric inequality.

Proposition 8. Suppose $T \in \mathbb{R}$ is large enough, so that the estimate of Lemma 2 holds. Let $T<t_{1}<\ldots<t_{d}$ and let $J_{\Phi_{\Gamma}}$ be the Jacobian of the mapping

$$
\Phi_{\Gamma}\left(t_{1}, \ldots, t_{d}\right)=\sum_{i=1}^{d} \Gamma\left(t_{i}\right)
$$

Then

$$
J_{\Phi_{\Gamma}}\left(t_{1}, \ldots, t_{d}\right) \gtrsim \prod_{i=1}^{d}\left|L_{\Gamma}\left(t_{i}\right)\right|^{\frac{1}{d}} \prod_{1 \leq i<j \leq d}\left(t_{j}-t_{i}\right)
$$

Proof. All the variables in this proof are taken to be greater than $T$. Let us define

$$
K_{m}=\prod_{i=1}^{m}\left(a_{i} \psi_{i}(\infty)\right) \prod_{1 \leq i<j \leq m}\left(a_{j}-a_{i}\right)
$$

so that the estimate of Lemma 2 now reads

$$
L_{\Gamma_{1} \cdots \Gamma_{m}}(t) \sim K_{m} e^{t \sum_{i=1}^{m} a_{i}} .
$$

In order to use (5), we need to calculate the various quantities $I_{r}$ for our curve $\Gamma$. We first show inductively for $1 \leq r \leq d-2$ that

$$
I_{r}\left(x_{1}, \ldots, x_{r}\right) \gtrsim \frac{K_{d-r-1}^{r} K_{d}}{K_{d-r}^{r+1}} \prod_{s=1}^{r} e^{x_{s}\left[\frac{1}{r}\left(\sum_{i=d-r+1}^{d} a_{i}\right)-a_{d-r}\right]} \prod_{1 \leq i<j \leq r}\left(x_{j}-x_{i}\right) .
$$

For $r=1$ we have

$$
I_{1}(x)=\frac{L_{\Gamma_{1} \ldots \Gamma_{d-2}}(x) L_{\Gamma}(x)}{L_{\Gamma_{1} \ldots \Gamma_{d-1}}(x)^{2}}=\frac{K_{d-2} K_{d}}{K_{d-1}^{2}} e^{x\left(a_{d}-a_{d-1}\right)} .
$$


Let us now suppose that (11) holds for some integer $r \in[1, d-3]$. Then

$$
\begin{aligned}
I_{r+1} & \left(x_{1}, \ldots, x_{r+1}\right) \\
= & \prod_{s=1}^{r+1} \frac{L_{\Gamma_{1} \ldots \Gamma_{d-r-2}}\left(x_{s}\right) L_{\Gamma_{1} \ldots \Gamma_{d-r}}\left(x_{s}\right)}{L_{\Gamma_{1} \ldots \Gamma_{d-r-1}}\left(x_{s}\right)^{2}} \int_{x_{1}}^{x_{2}} d y_{1} \ldots \int_{x_{r}}^{x_{r+1}} d y_{r} I_{r}\left(y_{1}, \ldots, y_{r}\right) \\
\gtrsim & \left(\frac{K_{d-r-2} K_{d-r}}{K_{d-r-1}^{2}}\right)^{r+1} \frac{K_{d-r-1}^{r} K_{d}}{K_{d-r}^{r+1}} \prod_{s=1}^{r+1} e^{x_{s}\left(a_{d-r}-a_{d-r-1}\right)} \\
& \times \int_{x_{1}}^{x_{2}} d y_{1} \ldots \int_{x_{r}}^{x_{r+1}} d y_{r} \prod_{s=1}^{r} e^{y_{s}\left[\frac{1}{r}\left(\sum_{i=d-r+1}^{d} a_{i}\right)-a_{d-r}\right]} \prod_{1 \leq i<j \leq r}\left(y_{j}-y_{i}\right) \\
\gtrsim & \frac{K_{d-r-2}^{r+1} K_{d}}{K_{d-r-1}^{r+2}} \prod_{s=1}^{r+1} e^{x_{s}\left[a_{d-r}-a_{d-r-1}+\frac{1}{r+1}\left(\sum_{i=d-r+1}^{d} a_{i}\right)-\frac{r}{r+1} a_{d-r}\right]} \prod_{1 \leq i<j \leq r+1}\left(x_{j}-x_{i}\right) \\
= & \frac{K_{d-r-2}^{r+1} K_{d}}{K_{d-r-1}^{r+2}} \prod_{s=1}^{r+1} e^{x_{s}\left[\frac{1}{r+1}\left(\sum_{i=d-r}^{d} a_{i}\right)-a_{d-r-1}\right]} \prod_{1 \leq i<j \leq r+1}\left(x_{j}-x_{i}\right),
\end{aligned}
$$

which is inequality (11) with $r+1$ instead of $r$. Here, we used Lemma 2 and Corollary 7 The expressions for $I_{d-1}$ and $I_{d}$ are slightly different, but simple calculations give

$$
I_{d-1}\left(x_{1}, \ldots, x_{d-1}\right) \gtrsim \frac{K_{d}}{K_{1}^{d}} \prod_{s=1}^{d-1} e^{x_{s}\left[\frac{1}{d-1}\left(\sum_{i=2}^{d} a_{i}\right)-a_{1}\right]} \prod_{1 \leq i<j \leq d-1}\left(x_{j}-x_{i}\right)
$$

and

$$
I_{d}\left(x_{1}, \ldots, x_{d}\right) \gtrsim K_{d} \prod_{s=1}^{d} e^{x_{s} \frac{1}{d} \sum_{i=1}^{d} a_{i}} \prod_{1 \leq i<j \leq d}\left(x_{j}-x_{i}\right),
$$

which is ultimately the inequality of interest here. As a consequence of this last inequality, we have

$$
\begin{aligned}
J_{\Phi_{\Gamma}}\left(t_{1}, \ldots, t_{d}\right) & =I_{d}\left(t_{1}, \ldots, t_{d}\right) \\
& \gtrsim K_{d} \prod_{s=1}^{d} e^{t_{s} \frac{1}{d} \sum_{i=1}^{d} a_{i}} \prod_{1 \leq i<j \leq d}\left(t_{j}-t_{i}\right) \\
& \sim \prod_{i=1}^{d}\left|L_{\Gamma}\left(t_{i}\right)\right|^{\frac{1}{d}} \prod_{1 \leq i<j \leq d}\left(t_{j}-t_{i}\right),
\end{aligned}
$$

concluding the proof of Proposition 8

\section{Higher ORDER OFFSPRING CURVES}

We shall call the offspring curves $\Gamma_{h}$, defined by Definition 3, first order offspring curves of $\Gamma$. We can then consider the family of offspring curves of $\Gamma_{h}$, for all first order offspring curves $\Gamma_{h}$, and in this way obtain second order offspring curves of $\Gamma$. Repeating this process we produce higher order offspring curves. The $n$ 'th order offspring curves of $\Gamma$ have the form

$$
\Gamma_{\eta}(t)=\left(\Gamma_{\eta, 1}, \ldots, \Gamma_{\eta, d}\right)=\sum_{j=1}^{J} \Gamma\left(t+\eta_{j}\right)
$$


where $J=d^{n}, \eta=\left(\eta_{1}, \ldots, \eta_{J}\right) \in \mathbb{R}^{J}$ and $0=\eta_{1} \leq \eta_{2} \leq \ldots \leq \eta_{J}$. Due to the exponential parametrisation of our original curve $\Gamma$, any offspring curve of $\Gamma$, of any order, resembles $\Gamma$. In particular,

$$
\gamma(t)=\left(\gamma_{1}(t), \ldots, \gamma_{d}(t)\right):=\Gamma_{\eta}(t)=\sum_{j=1}^{J} \Gamma\left(t+\eta_{j}\right)
$$

satisfies

$$
\gamma_{i}(t)=e^{a_{i} t} \sum_{j=1}^{J} e^{a_{i} \eta_{j}} \psi_{i}\left(t+\eta_{j}\right),
$$

for each $1 \leq i \leq d$. Therefore, if we let $\varphi_{i}(t)=\sum_{j=1}^{J} e^{a_{i} \eta_{j}} \psi_{i}\left(t+\eta_{j}\right)$, we have

$$
\lim _{t \rightarrow \infty} \varphi_{i}(t)=\psi_{i}(\infty) \sum_{j=1}^{J} e^{a_{i} \eta_{j}}=: \varphi_{i}(\infty)
$$

and, for all $1 \leq m \leq d$,

$$
\lim _{t \rightarrow \infty} \varphi_{i}^{(m)}(t)=0
$$

In addition, we have the following generalisation of Lemmas 2 and 4. Let us define, for $1 \leq m \leq d$ and $\eta$ as above,

$$
L_{\eta, \Gamma_{1} \ldots \Gamma_{m}}(t)=\left|\begin{array}{ccc}
\Gamma_{\eta, 1}^{\prime}(t) & \cdots & \Gamma_{\eta, 1}^{(m)}(t) \\
\vdots & & \vdots \\
\Gamma_{\eta, m}^{\prime}(t) & \cdots & \Gamma_{\eta, m}^{(m)}(t)
\end{array}\right| .
$$

Lemma 9. Let $\Gamma_{\eta}$ be an n'th generation offspring curve of $\Gamma, J=d^{n}$, and $1 \leq$ $m \leq d$. Then, there exists $T$, depending on $d$, the $a_{i}$, and the $\psi_{i}$, such that

$$
L_{\eta, \Gamma_{1} \ldots \Gamma_{m}}(t) \sim e^{t \sum_{i=1}^{m} a_{i}} \prod_{i=1}^{m}\left(a_{i} \psi_{i}(\infty) \sum_{j=1}^{J} e^{a_{i} \eta_{j}}\right) \prod_{1 \leq i<j \leq m}\left(a_{j}-a_{i}\right)
$$

for all $t>T$.

Note that, as in Lemma 4, the implicit constants in (12) can be chosen absolutely from the outset and in particular do not depend on $d$, the $a_{i}$, the $\psi_{i}$ or $T$. We omit the proof of this result, since it is an exact copy of the proof of Lemma 4 if one replaces the number of summands $d$ in the definition of $\Gamma_{h}$ by $J, h$ by $\eta$ and the block size $d$ of the corresponding matrices by $m \leq d$. As before, it is important here that the $\eta_{j}$ are nonnegative, so that $t>T$ also implies $t+\eta_{j}>T$.

Note that because of Lemma 9 we may pick the same $T$ for all offspring curves of $\Gamma$ of all orders.

\section{Proof of Theorem 1}

Let us define the measure $d \sigma_{T, R}$, by its action on test functions $\phi: \mathbb{R}^{d} \rightarrow \mathbb{R}$, as

$$
d \sigma_{T, R}(\phi)=\int_{T}^{R} \phi(\Gamma(t))\left|L_{\Gamma}(t)\right|^{\frac{2}{d(d+1)}} d t
$$

and its one-dimensional analogue $d \omega_{T, R}$, by its action on test functions $\phi: \mathbb{R} \rightarrow \mathbb{R}$, as

$$
d \omega_{T, R}(\phi)=\int_{T}^{R} \phi(t)\left|L_{\Gamma}(t)\right|^{\frac{2}{d(d+1)}} d t .
$$


Using the exponential reparametrisation, it suffices to prove the dual inequality to (3), which in return is equivalent to proving that

$$
\left\|\widehat{g d \sigma_{T, R}}\right\|_{L^{q}\left(B_{r}\right)} \leq C_{p}\|g\|_{L^{p}\left(d \omega_{T, R}\right)}
$$

for all Schwartz functions $g$,

$$
\frac{1}{p}+\frac{d(d+1)}{2 q}=1, \quad 1 \leq p<\frac{d^{2}+d+2}{2}=: D
$$

uniformly in $R$ and all balls $B_{r}$ of radius $r$ and centre at the origin, and a fixed large enough $T$, which will be chosen later. Clearly, $\delta$ in Theorem 1 will be set equal to $e^{-T}$.

Let $\mathcal{K}_{T}$ denote the class of all curves of the form (44) with $a_{i}$ and $\psi_{i}$ satisfying the conditions set out in the beginning of Section 2 (i.e. that $a_{1}<\ldots<a_{d}$, for each $1 \leq i \leq d, \psi_{i}(\infty):=\lim _{t \rightarrow \infty} \psi_{i}(t)$ exists and is nonzero and $\lim _{t \rightarrow \infty} \psi_{i}^{(m)}(t)=0$ for all $1 \leq m \leq d$ ) and that satisfy (12) for all $t>T$. The constants implicit in the $\sim$ symbol in (12) can be fixed from the start (e.g. 1/2 and 3/2) and are not important. Because of Lemma 9, every curve of the above form belongs to some class $\mathcal{K}_{T}$ for some large enough $T$. Moreover, all of its offspring curves of any order belong to the same class $\mathcal{K}_{T}$. Let us now define

$$
\mathcal{A}(T, R, r)=\sup _{\Gamma \in \mathcal{K}_{T}}\left(\sup _{\|g\|_{L^{p}\left(d \omega_{T, R}\right)} \leq 1} \| \widehat{\left.g d \sigma_{T, R} \|_{L^{q}\left(B_{r}\right)}\right)} .\right.
$$

It is easy to see that $\mathcal{A}(T, R, r)<\infty$ for every $T$ sufficiently large, $R$ and $r$, but it is our goal to prove a bound for $\mathcal{A}(T, R, r)$ which is uniform in $T, R$, and $r$.

Given any $\Gamma \in \mathcal{K}_{T}$, we consider the $d$-fold product

$$
\begin{aligned}
\prod_{i=1}^{d} \widehat{g_{i} \widehat{d \sigma_{T, R}}} & =\int_{T}^{R} \ldots \int_{T}^{R} e^{i x \cdot \sum_{i=1}^{d} \Gamma\left(t_{i}\right)} \prod_{i=1}^{d}\left(g_{i}\left(t_{i}\right)\left|L_{\Gamma}\left(t_{i}\right)\right|^{\frac{2}{d(d+1)}}\right) d t_{1} \ldots d t_{d} \\
& =\sum_{\pi \in S_{d}} \int_{D_{T, R, \pi}} e^{i x \cdot \sum_{i=1}^{d} \Gamma\left(t_{i}\right)} \prod_{i=1}^{d}\left(g_{i}\left(t_{i}\right)\left|L_{\Gamma}\left(t_{i}\right)\right|^{\frac{2}{d(d+1)}}\right) d t_{1} \ldots d t_{d}
\end{aligned}
$$

where $S_{d}$ denotes the group of permutations of $\{1, \ldots, d\}$ and $D_{T, R, \pi}=\left\{\left(t_{1}, \ldots, t_{d}\right)\right.$ $\left.\in[T, R]^{d}: t_{\pi(1)}<\ldots<t_{\pi(d)}\right\}$. For notational convenience, here we are using a different sign convention than usual for the Fourier transform. Let us now fix a $\pi \in S_{d}$ and consider the corresponding term in the above sum. We perform the change of variables $t_{\pi(1)}=t$ and $t_{\pi(i)}=t+h_{i}$, for $2 \leq i \leq d$, in order to obtain

$$
\begin{aligned}
& \int_{D_{T, R, \pi}} e^{i x \cdot \sum_{i=1}^{d} \Gamma\left(t_{i}\right)} \prod_{i=1}^{d}\left(g_{i}\left(t_{i}\right)\left|L_{\Gamma}\left(t_{i}\right)\right|^{\frac{2}{d(d+1)}}\right) d t_{1} \ldots d t_{d} \\
& \quad=\int_{\Delta_{T, R}}\left(\int_{T}^{R-h_{d}} e^{i x \cdot \Gamma_{h}(t)} \prod_{i=1}^{d}\left(g_{i}\left(t+h_{i}\right) \mid L_{\Gamma}\left(t+h_{i}\right)^{\frac{2}{d(d+1)}}\right) d t\right) d h^{\prime},
\end{aligned}
$$


where $\Delta_{T, R}=\left\{h^{\prime}=\left(h_{2}, \ldots, h_{d}\right) \in \mathbb{R}^{d-1}: 0<h_{2}<\ldots<h_{d-1}<h_{d}<R-T\right\}$, and where $h_{1}=0$. Since $\Gamma_{h} \in \mathcal{K}_{T}$, we have

$$
\begin{aligned}
& \left(\int_{B_{r}}\left|\int_{\Delta_{T, R}} \int_{T}^{R-h_{d}} e^{i x \cdot \Gamma_{h}(t)} \prod_{i=1}^{d}\left(g_{i}\left(t+h_{i}\right)\left|L_{\Gamma}\left(t+h_{i}\right)\right|^{\frac{2}{d(d+1)}}\right) d t d h^{\prime}\right|^{q} d x\right)^{\frac{1}{q}} \\
& \leq \mathcal{A}(T, R, r) \\
& \times \int_{\Delta_{T, R}}\left(\left.\left.\int_{T}^{R-h_{d}} \prod_{i=1}^{d}\left|g_{i}\left(t+h_{i}\right)\right| L_{\Gamma}\left(t+h_{i}\right)\right|^{\frac{2}{d(d+1)}}\right|^{p}\left|L_{\Gamma_{h}}(t)\right|^{\frac{2(1-p)}{d(d+1)}} d t\right)^{\frac{1}{p}} d h^{\prime} .
\end{aligned}
$$

We may also use Plancherel's theorem together with the change of variables

$$
\left(t, h^{\prime}\right) \mapsto \Phi_{\Gamma}\left(t, h^{\prime}\right)=\Gamma_{h}(t)
$$

to obtain

$$
\begin{aligned}
& \left(\int_{B_{r}}\left|\int_{\Delta_{T, R}} \int_{T}^{R-h_{d}} e^{i x \cdot \Gamma_{h}(t)} \prod_{i=1}^{d}\left(g_{i}\left(t+h_{i}\right)\left|L_{\Gamma}\left(t+h_{i}\right)\right|^{\frac{2}{d(d+1)}}\right) d t d h^{\prime}\right|^{2} d x\right)^{\frac{1}{2}} \\
& \lesssim\left(\left.\left.\int_{\Delta_{T, R}} \int_{T}^{R-h_{d}} \prod_{i=1}^{d}\left|g_{i}\left(t+h_{i}\right)\right| L_{\Gamma}\left(t+h_{i}\right)\right|^{\frac{2}{d(d+1)}}\right|^{2}\left|J_{\Gamma}\left(t, h^{\prime}\right)\right|^{-1} d t d h^{\prime}\right)^{\frac{1}{2}}
\end{aligned}
$$

where $J_{\Gamma}$ is the Jacobian of the mapping $\Phi_{\Gamma}$. The fact that $\Phi_{\Gamma}$ is a one-to-one mapping for $t>T, h^{\prime} \in \Delta_{T, R}$, follows from Proposition 8 together with an argument that goes back to Steinig [14 and has been presented in a number of articles (see e.g. [11, Section 3]). In [7] a variant of this argument was presented where one may only assume that the minors of $L_{\Gamma}$ are single-signed (see Proposition 6.1 in that article).

By analytic interpolation, using (15) and (16) (see [3]), we have that

$$
\begin{aligned}
& \left(\int_{B_{r}}\left|\int_{\Delta_{T, R}} \int_{T}^{R-h_{d}} e^{i x \cdot \gamma_{h}(t)} \prod_{i=1}^{d}\left(g_{i}\left(t+h_{i}\right)\left|L_{\Gamma}\left(t+h_{i}\right)\right|^{\frac{2}{d(d+1)}}\right) d t d h^{\prime}\right|^{c} d x\right)^{\frac{1}{c}} \\
& \lesssim \mathcal{A}(T, R, r)^{1-\theta}\left(\int _ { \Delta _ { T , R } } \left(\left.\left.\int_{T}^{R-h_{d}} \prod_{i=1}^{d}\left|g_{i}\left(t+h_{i}\right)\right| L_{\Gamma}\left(t+h_{i}\right)\right|^{\frac{2}{d(d+1)}}\right|^{b}\right.\right. \\
& \left.\left.\left|J_{\Gamma}\left(t, h^{\prime}\right)\right|^{b\left(\frac{1}{a}-1\right)}\left|L_{\Gamma_{h}}(t)\right|^{\frac{2}{d(d+1)}}\left(1-\frac{b}{a}\right) d t\right)^{\frac{a}{b}} d h^{\prime}\right)^{\frac{1}{a}}
\end{aligned}
$$

where

$$
0 \leq \theta \leq 1, \quad \frac{1}{a}=1-\frac{\theta}{2}, \quad \frac{1}{b}=\frac{1}{p}+\theta\left(\frac{1}{2}-\frac{1}{p}\right), \quad \frac{1}{c}=\frac{1}{q}+\theta\left(\frac{1}{2}-\frac{1}{q}\right)
$$


Notice that $b \geq a$. Applying Propositions 5 and 8 , we have

$$
\begin{aligned}
& \left(\int_{B_{r}}\left|\int_{\Delta_{T, R}} \int_{T}^{R-h_{d}} e^{i x \cdot \gamma_{h}(t)} \prod_{i=1}^{d}\left(g_{i}\left(t+h_{i}\right)\left|L_{\Gamma}\left(t+h_{i}\right)\right|^{\frac{2}{d(d+1)}}\right) d t d h^{\prime}\right|^{c} d x\right)^{\frac{1}{c}} \\
& \lesssim \mathcal{A}(T, R, r)^{1-\theta}\left(\int _ { \Delta _ { T , R } } \left(\int_{T}^{R-h_{d}} \prod_{i=1}^{d}\left|g_{i}\left(t+h_{i}\right)\right|^{b}\right.\right. \\
& \left.\left.\left|L_{\Gamma}\left(t+h_{i}\right)\right|^{\frac{2 b}{d(d+1)}+\frac{b}{d}\left(\frac{1}{a}-1\right)+\frac{2 b}{d^{2}(d+1)}\left(\frac{1}{b}-\frac{1}{a}\right)} d t\right)^{\frac{a}{b}}\left(\prod_{i=2}^{d} h_{i} \prod_{2 \leq i<j \leq d}\left(h_{j}-h_{i}\right)\right)^{1-a} d h^{\prime}\right)^{\frac{1}{a}} .
\end{aligned}
$$

The rest of the argument is now similar to the one in Section 6 of [2. We need to choose $\theta$ such that the exponent

$$
\begin{aligned}
\frac{2 b}{d(d+1)}+\frac{b}{d}\left(\frac{1}{a}-1\right)+\frac{2 b}{d^{2}(d+1)}\left(\frac{1}{b}-\frac{1}{a}\right) & =\frac{b}{d}\left[\frac{2}{d+1}-\frac{\theta}{2}-\frac{2(1-\theta)}{d(d+1) p^{\prime}}\right] \\
& =\frac{2 b}{d(d+1) p} .
\end{aligned}
$$

This requires the choice

$$
\theta=\frac{4(d-1)}{d(d+1) p^{\prime}-4}
$$

Let us denote by $L^{r, s}$ the Lorentz space on $\mathbb{R}$ and by $\|\cdot\|_{r, s}$ the corresponding norm. We may now apply Theorem 5 of [10] (see also Proposition 2.4(ii) of [2]) to obtain

$$
\left\|\prod_{i=1}^{d} \widehat{g_{i} \widehat{d \sigma_{T, R}}}\right\|_{L^{q / d}\left(B_{r}\right)} \leq C_{p} \mathcal{A}(T, R, r)^{1-\theta} \prod_{i=1}^{d}\left\|g_{i}\left|L_{\Gamma}\right|^{\frac{2}{d(d+1) p}}\right\|_{p_{i}, 1}
$$

for all $\left(p_{1}^{-1}, \ldots, p_{d}^{-1}\right) \in \Sigma(a, b)$, where from now on $a$ and $b$ take the values corresponding to the value of $\theta$ given by (17) and $\Sigma(a, b)$ denotes the $(d-1)$-dimensional closed convex hull of the points $Q_{i}, 1 \leq i \leq d$, where the $i$ 'th coordinate of each $Q_{i}$ is equal to $1 / b$ and the rest of the coordinates are equal to $(d+2-d a) /(2 a)$. Note that the use of this result requires the restriction $p<D$. We then consider the $D$-linear operator (note that $D$, defined in (14), is always an integer)

$$
\mathcal{M}_{T, R}\left(\psi_{1}, \ldots, \psi_{D}\right)=\prod_{i=1}^{D}\left(\psi_{i}\left|L_{\Gamma}\right|^{-\frac{2}{d(d+1) p}} d \sigma_{T, R}\right)
$$

and the set $Y$ of all points $P^{\pi}=\left(P_{1}^{\pi}, \ldots, P_{D}^{\pi}\right)$, where $\pi \in S_{D}$ (the set of permutations of $\{1, \ldots, D\})$ and

$$
P_{\pi(i)}^{\pi}=\left\{\begin{array}{cl}
1 / b, & i=1 \\
(d+2-d a) /(2 a), & 2 \leq i \leq d \\
1 / p, & d+1 \leq i \leq D .
\end{array}\right.
$$


By (18) and the multilinear Hölder's inequality we have

$$
\left\|\mathcal{M}_{T, R}\left(\psi_{1}, \ldots, \psi_{D}\right)\right\|_{L^{q / D}\left(B_{r}\right)} \leq C_{p} \mathcal{A}(T, R, r)^{D-d+1-\theta} \prod_{i=1}^{D}\left\|\psi_{i}\right\|_{p_{i}, 1}
$$

for all $\left(p_{1}^{-1}, \ldots, p_{D}^{-1}\right) \in Y$. We may now apply Christ's multilinear interpolation (see Proposition 2.3 in 2]) to obtain

$$
\left\|\mathcal{M}_{T, R}\left(\psi_{1}, \ldots, \psi_{D}\right)\right\|_{L^{q / D}\left(B_{r}\right)} \leq C_{p} \mathcal{A}(T, R, r)^{D-d+1-\theta} \prod_{i=1}^{D}\left\|\psi_{\pi(i)}\right\|_{p_{i}, D}
$$

for all $\left(p_{1}^{-1}, \ldots, p_{D}^{-1}\right) \in(\operatorname{conv} Y)^{o}$. Since $\left(p^{-1}, \ldots, p^{-1}\right) \in(\operatorname{conv} Y)^{o}$, we have for $\psi_{i}=g\left|L_{\Gamma}\right|^{\frac{2}{d(d+1) p}} \chi_{[T, R]}, 1 \leq i \leq D$,

$$
\begin{aligned}
\left\|\widehat{g d \sigma_{T, R}}\right\|_{L^{q}\left(B_{r}\right)} & =\left\|\mathcal{M}_{T, R}\left(\psi_{1}, \ldots, \psi_{D}\right)\right\|_{L^{q / D}\left(B_{r}\right)}^{1 / D} \\
& \leq C_{p} \mathcal{A}(T, R, r)^{(D-d+1-\theta) / D}\left\|\left.g L_{\Gamma}\right|^{\frac{2}{d(d+1) p}} \chi_{[T, R]}\right\|_{p, D} \\
& \leq C_{p} \mathcal{A}(T, R, r)^{(D-d+1-\theta) / D}\left\|\left.g L_{\Gamma}\right|^{\frac{2}{d(d+1) p}} \chi_{[T, R]}\right\|_{p} \\
& =C_{p} \mathcal{A}(T, R, r)^{1-(d-1+\theta) / D}\|g\|_{L^{p}\left(d \omega_{T, R}\right)}
\end{aligned}
$$

Taking the supremum over all curves $\Gamma \in \mathcal{K}_{T}$, we have

$$
\mathcal{A}(T, R, r)^{(d-1+\theta) / D} \leq C_{p},
$$

which shows (13) and completes the proof of Theorem 1

\section{REFERENCES}

1. G. I. Arkhipov, V. N. Chubarikov, and A. A. Karatsuba, Exponent of convergence of the singular integral in the Terry problem, (Russian) Dokl. Akad. Nauk SSSR 248 (1979), no. 2, 268-272. MR553185 (81h:10058)

2. J.-G. Bak, D. Oberlin, and A. Seeger, Restriction of Fourier transforms to curves and related oscillatory integrals, Amer. J. Math. 131 (2009), no. 2, 277-311. MR2503984 (2010b:42012)

3. A. Benedek and R. Panzone, The spaces $L^{p}$, with mixed norm, Duke Math. J. 28 (1961), 301-324. MR0126155(23:A3451)

4. S. Dendrinos, Generalisations of an inequality of Hardy under polynomial changes of variables, Ph.D. thesis, 2005, University of Edinburgh.

5. S. Dendrinos, On the restriction of the Fourier transform to polynomial curves, Complex and Harmonic Analysis: Proceedings of the International Conference, May 25-27, 2006, Aristotle University of Thessaloniki, 273-294, DEStech Publications, Lancaster PA, 2007. MR2387296 (2009h:42010)

6. S. Dendrinos, Fourier restriction of $H^{1}$ functions on polynomial surfaces, J. Fourier Anal. Appl. 13 (2007), no. 5, 623-641. MR2355016(2008h:42038)

7. S. Dendrinos and J. Wright, Fourier restriction to polynomial curves I: a geometric inequality, Amer. J. Math. 132 (2010), no. 4, 1031-1076. MR2663647 (2011e:42035)

8. S. W. Drury, Restriction of Fourier transforms to curves, Ann. Inst. Fourier, 35 (1985), no. 1, 117-123. MR781781 (86e:42026)

9. S. W. Drury, Degenerate curves and harmonic analysis, Math. Proc. Cambridge Philos. Soc. 108 (1990), no. 1, 89-96. MR.1049762 (91h:42021)

10. S. W. Drury and B. P. Marshall, Fourier restriction theorems for curves with affine and Euclidean arclengths, Math. Proc. Cambridge Philos. Soc. 97 (1985), no. 1, 111-125. MR764500 (87b:42019)

11. S. W. Drury and B. P. Marshall, Fourier restriction theorems for degenerate curves, Math. Proc. Cambridge Philos. Soc. 101 (1987), no. 3, 541-553. MR878901 (88e:42026)

12. B. Shayya, An affine restriction estimate in $\mathbb{R}^{3}$, Proc. Amer. Math. Soc. 135 (2007), no. 4, 1107-1113. MR 2262912(2007k:42020) 
13. P. Sjölin, Fourier multipliers and estimates for the Fourier transform of measures carried by smooth curves in $\mathbb{R}^{2}$, Studia Math. 51 (1974), 169-182. MR0385437 (52:6299)

14. J. Steinig, On some rules of Laguerre's and systems of equal sums of like powers, Rend. Math. 6 (1971), no. 4, 629-644. MR0309867 (46:8972)

Department of Mathematics and Statistics, University of Jyväskylä, P.O. Box 35 (MAD), 40014 JyVÄSKYlä, Finland

E-mail address: spyridon.dendrinos@jyu.fi

Current address: Department of Mathematics and Statistics, University of Helsinki, P.O. Box 68 (Gustaf Hällströmin katu 2b), 00014 Helsinki, Finland

E-mail address: spyridon.dendrinos@helsinki.fi

Mathematisches Seminar, C. A.-Universität Kiel, Ludewig-Meyn-Strasse 4, 24098 Kiel, Germany

E-mail address: mueller@math.uni-kiel.de 